[1]

'Oxford Pleural Unit, Churchill Hospital, Oxford, UK.

2Oxford Respiratory Trials Unit, Churchill Hospital, Oxford, UK.

${ }^{3}$ Oxford University Hospitals NHS Foundation Trust, Oxford, UK.

\title{
Secondary pneumothorax in end-stage lung disease complicated by noninvasive ventilation and a persistent air leak
}

\section{Case report}

A 75-year-old man initially presented to the respiratory service in 2012 with severe chronic obstructive pulmonary disease (COPD) defined as Global Initiative for Chronic Obstructive Lung Disease stage 3. Forced expiratory volume in $1 \mathrm{~s}\left(\mathrm{FEV}_{1}\right)$ was $1.1 \mathrm{~L}$ (33\% predicted) with $\mathrm{FEV}_{1}$ / forced vital capacity 32\%. He required long-term oxygen therapy at home at $4 \mathrm{~L} \cdot \mathrm{min}^{-1}$ and by 2015 nocturnal high-pressure noninvasive ventilation
(NIV) was required due to progressive symptomatic hypercapnic respiratory failure including a carbon dioxide tension $\left(\mathrm{PCO}_{2}\right)$ of $7.9 \mathrm{kPa}$ and bicarbonate of $28.3 \mathrm{mmol} \cdot \mathrm{L}^{-1}$.

\section{Task 1}

What is the role of long-term NIV in hypercapnic respiratory failure secondary to COPD?
Cite as: McCracken DJ, Bedawi EO, Hassan M, et al. Secondary pneumothorax in end-stage lung disease complicated by noninvasive ventilation and a persistent air leak. Breathe 2018; 14: e119-e122. 


\section{Answer 1}

Recurrent exacerbations are characteristic of COPD and may lead to respiratory failure requiring ventilatory support. The use of NIV in this setting became an established treatment for acute exacerbations in the early 2000s; however, clinical trials such as the RESCUE trial, initially failed to demonstrate improvements in hypercapnia or mortality with domiciliary use [1]. The HOT-HMV trial [2], published in 2017, demonstrated statistically significant improvements in time to readmission or death in patients treated with oxygen and NIV at home when compared with those treated with oxygen alone, which in contrast to previous studies justified its use. There were also reductions in frequency of exacerbations and improvements in health-related quality of life scores at 3 months but no difference in all-cause mortality [2]. Use of domiciliary NIV in COPD therefore remains contentious, but would appear to result in symptomatic benefit in a subset of patients along with probable reductions in readmission rates.

The patient was admitted in December 2017 with a large symptomatic left-sided secondary spontaneous pneumothorax causing respiratory compromise and surgical emphysema. Initial management was with an $18 \mathrm{Fr}$ apical chest drain inserted in the emergency department with good symptomatic and radiological improvement. A persistent air leak remained and NIV was temporarily discontinued.

Task 2

What size of intercostal chest drain is appropriate for the initial management of a secondary spontaneous pneumothorax?

\section{Answer 2}

The British Thoracic Society pleural disease guidelines (2010) suggest first line use of smallbore chest tubes $(<14 \mathrm{Fr})$ in pneumothoraces requiring drainage [3]. This is largely based on the perceived increase in pain associated with larger drains, along with the risk of serious complications such as malposition, visceral injury or infective complications such as empyema. The documented efficacy of smallbore drains is adequate to suggest their use, and while many of the studies directly comparing the use of small-bore and large-bore chest drains are limited by their retrospective nature and small patient numbers, none demonstrate any statistically significant difference in efficacy. Expert consensus would therefore suggest that small-bore drains should generally be used in pneumothorax management, but that it may be necessary to consider a largebore drain in cases of a blocked small drain resulting in tension pneumothorax, or in cases where an air leak is not adequately controlled despite the use of thoracic suction.

After 1 week of inpatient management, an air leak persisted with incomplete apposition of the lung. The patient was deemed unfit for surgical intervention and therefore a Heimlich valve was applied to allow for ambulatory, outpatient management. Over 4 weeks, the patient attended for weekly review. Functional capacity increased and the air leak was measured objectively as 150$180 \mathrm{~mL} \cdot \mathrm{min}^{-1}$ using a digital suction device (Thopaz; Medela UK Ltd, Manchester, UK).

The patient developed early morning headaches and daytime somnolence with $\mathrm{PCO}_{2}$ rising to $8.5 \mathrm{kPa}$. NIV was recommenced with chest radiography and Thopaz reassessment to ensure no worsening pneumothorax. Improvements in $\mathrm{PCO}_{2}$ and symptoms resulted, radiological appearances were unchanged and objective air leak remained at 180-200 $\mathrm{mL} \cdot \mathrm{min}^{-1}$

Task 3

How does long-term NIV use influence pneumothorax management and what methods are available to assess this objectively? 


\section{Answer 3}

Thopaz is a digital suction device that allows for accurate objective air leak measurement in pneumothorax management. It is a novel option to guide management and may have a role in risk stratification. This role is currently under investigation in the ongoing randomised ambulatory management of primary pneumothorax (RAMPP) trial (https:// doi.org/10.1186/ISRCTN79151659) in order to assess whether a large air leak can predict those patients whose pneumothorax is unlikely to resolve and therefore require earlier surgical intervention.

The effect of NIV on a persistent air leak is unclear with no evidence to suggest impaired resolution; physiologically, it is likely that the positive airway pressure is significantly attenuated and not transmitted to the pleural surface and peripheral lung parenchyma. However, NIV was temporarily discontinued due to the perceived association between positive pressure ventilation and barotrauma or volutrauma. In this case, objectively measured air leak was unchanged irrespective of ventilator use and following a symptomatic deterioration, NIV was recommenced.

To facilitate the patient's wishes for drain removal, he was readmitted for blood patch pleurodesis which was performed on three occasions but was unsuccessful. Air leak persisted at $>100 \mathrm{~mL} \cdot \mathrm{min}^{-1}$ so the patient was discharged with a Heimlich valve for further ambulatory follow-up. At the time of writing, 5 months after initial presentation, the patient remains under regular ongoing follow-up with a persistent air leak.

\section{Task 4}

What options are available in the management of patients with a persistent air leak?

\section{Answer 4}

Several methods are available in the management of persistent air leak including a surgical approach, endobronchial valve placement, medical pleurodesis or ambulatory management. In some cases, transplant could also be considered, although in this case age and deconditioning were significant relative contraindications. A number of pleurodesis agents are available, with talc the most commonly used in the UK, as it is recognised as being relatively safe with an apparently low pneumothorax recurrence rate. Evidence underpinning the relative success rates between agents, however, is limited by the paucity of quality data [4].

Autologous blood can be used and is the pleurodesis agent of choice for the authors in cases of persistent air leak such as this, due to a previous case of severe pneumonitis resulting in the death of the patient following talc administration. The suggested method of action is also thought to be different with the theoretical sealing of the air leak as opposed to the conventional adhesion of visceral and parietal pleura. Two reviews have suggested improvements when blood pleurodesis is compared with prolonged conservative management [5]. A theoretical risk exists with regards to pleural infection; however, only a single case of subsequent pleural infection has been identified from published case series [6].

There are few absolute contraindications to a surgical approach; however, the primary contraindications arise from anaesthetic risk and the requirement to maintain single lung ventilation throughout the procedure. Elective patients with a higher than average risk require a multidisciplinary approach including optimisation by the "pre-habilitation" nurses and an anaesthetic assessment which may result in further investigations to fully define this operative risk. In these cases, patients are informed of the increased risk of morbidity and mortality including the potential to worsen their quality of life and must understand and accept this before surgery will be offered. 
Both surgery and endobronchial valve placement were ultimately excluded in this case due to the significant associated peri-operative anaesthetic risk, predominantly as a result of this patient's severe hypercapnic respiratory failure. Surgery would also be technically difficult and would carry a higher risk of post-operative prolonged air leak given the extent of the emphysematous changes seen on computed tomography and the subsequent lack of normal lung tissue on which to place the resection line.

\section{Key points}

- The role of domiciliary positive pressure ventilation in patients with progressive hypercapnic respiratory failure remains unclear; however, recent evidence appears to favour its use.

- Pneumothorax is a well-recognised complication of end-stage COPD, but the management is often complex and may be complicated by other sequelae of advanced respiratory disease.

- A range of treatment options are available including various pleurodesis agents, but management must be tailored to the individual.

\section{Author contributions}

All authors contributed to the design and conception of the article. D.J. McCracken drafted the first manuscript. All authors were involved in management of the patient and all reviewed and approved the final manuscript.

\section{Conflict of interest}

None declared.

\section{References}

1. Struik FM, Sprooten RT, Kerstjens HA et al. Nocturnal non-invasive ventilation in COPD patients with prolonged hypercapnia after ventilatory support for acute respiratory failure: a randomised, controlled, parallel-group study. Thorax 2014; 69: 826-834

2. Murphy PB, Rehal S, Arbane G, et al. Effect of home noninvasive ventilation with oxygen therapy versus oxygen therapy alone on hospital readmission or death after an acute COPD exacerbation: a randomized clinical trial. JAMA 2017; 317: 2177-2186.

3. British Thoracic Society Pleural Disease Guideline Group. BTS pleural disease guideline 2010. Thorax 2010; 65: Suppl. 2, ii1-ii76.
4. Hallifax RJ, Yousuf $A$, Jones $H E$, et al. Effectiveness of chemical pleurodesis in spontaneous pneumothorax recurrence prevention: a systematic review. Thorax 2017; 72: 1121-1131.

5. Rinaldi S, Felton T, Bentley A. Blood pleurodesis for the medical management of pneumothorax. Thorax 2009; 64: 258-260.

6. Robinson CL. Autologous blood for pleurodesis in recurrent and chronic spontaneous pneumothorax. Can J Surg 1987; 30: 428-429. 